https://doi.org/10.48009/1_iis_2010_711-714

\title{
THE REVOLUTION OF USING COLLABORATIVE SOURCES IN HIGHER EDUCATION
}

\author{
Christine M. Shull, Central Michigan University, shull1cm@cmich.edu \\ Dana K. McCann, Central Michigan University, mccan1d@ cmich.edu
}

\begin{abstract}
Currently, there is a heated debate in academia concerning the reliability of Wikipedia in college research. Many professors and instructors question the source's credibility, and some discredit Wikipedia entirely. Unfortunately many students do not understand the open-source format of Wikipedia which could allow incomplete or even inaccurate information to be posted. However, it is also true that Wikipedia has easily accessible and often very relevant overview information that may be valuable as a preliminary source for students. Even though some professors and colleges have chosen to ban Wikipedia citations in their student's research, many experts question the wisdom of that policy because the very nature of information gathering has been forever changed by Internet collaborative sources. Instructors at the higher education level will be more effective when they allow the Internet as a source for knowledge and they give up the preconceived notion that Wikipedia and Internet sources are unreliable.
\end{abstract}

Keywords: Wikipedia, Academic Research, Collaborative Sources, Online Encyclopedia, Open Source, Credibility

\section{INTRODUCTION}

The dispute over Wikipedia's reliability as an academic source stems from its wiki structure. "Wiki" means quick, and that term is very disturbing to those in academia whom have always expected research to be edited and carefully checked before publication.

The debate concerning the use and value of Wikipedia in college research remains an important and complex issue. In fact, it could be argued that a discussion with students about the nature and reliability of Wikipedia presents a very good first

Volume XI, No. 1, 2010 step in teaching students how to use and evaluate research sources in every subject area.

\section{WIKIPEDIA IN ACADEMIA}

Wikipedia fully discloses statistics and facts about how knowledge is presented on its website. Since 2001 Wikipedia has become one of the most visited reference sites with over 78 million visitors monthly. There are more than " 91,000 active contributors working on more than $16,000,000$ articles in more than 270 languages." [4] The editing process is open to anyone with access to a computer who wishes to contribute to the articles.

Wikipedia admits that this open collaboration means that newer articles may "contain significant misinformation, unencyclopedic content, or vandalism." [4] It is important that students are aware that the editing process of Wikipedia is fluid and articles are subject to constant revision.

The ultimate goal is that entries will eventually be revised by expert scholars and experienced editors, and the "wiki" process will lead to quality, consensus and neutrality. A 2005 study in Nature found that when Wikipedia was compared to the Encyclopedia Britannica there was an average of three omissions in the edited formal encyclopedia compared to four in Wikipedia. [2] The Library Journal in 2006 reported that Wikipedia was especially strong and reliable in current affairs, pop culture, and science. Interestingly, the National Institute of Health presented a workshop to professionals on how to edit Wikipedia articles to keep the public updated and informed on health issues. [2]

There are signs that Wikipedia is gaining a foothold in academic circles. According to a new nationwide survey by the Pew Internet, fifty percent of doctors turn to Wikipedia for medical information and more than half of adults in the United States turn to the
Issues in Information Systems 
Internet when they have medical questions. [1] It is not surprising then that students also turn to the Internet when they begin to research a topic. In a rush to ban Wikipedia because of its admitted weaknesses instructors could miss a very effective teaching tool. They could also miss finding out just how much their students already know about research.

\section{LITERATURE REVIEW}

Sudin and Francke have done an extensive literature review and ethnographic study on the use of Wikipedia in the upper secondary classroom. They note that teachers and librarians no longer have control over the sources that students use in their formative years before entering college. [3] In the past students were dependent on school resources and classroom textbooks to complete research projects. Today's students are learning at an early age to access and accept Internet sources for information. The reality is that Wikipedia is flexible in many research areas, easy to access on networked computers, and familiar to students who have used the source at home and school. The traditional definitions schools and colleges have set to define and control citations in research will be increasingly challenged as the revolution in information technology continues.

Sudin and Francke also emphasize that researchers have studied the habits of students as they use these sources. They have concluded that students apply many of the traditional standards for judging sources that have always been considered good practices in researching sources. Wilson established in his studies that when students access a website they are already making research judgments about the validity of the information. [3] They consider the creator of the website, or if the creator is not evident they judge the reputation of the journals or organizations connected to the website. Students also note the style and tone as well as the documentation of the information. These habits are skills that help bring students to the college classroom ready to mature and become even more critical and adept in their research work.

\section{The Benefits of Using Wikipedia in College Research}

Websites like Wikipedia are not only familiar tools to students; but these tools influence the way student think, learn, and retain information. Sudin and Francke contend that "information seeking, credibility judgments and other information practices are treated as shaped within communities while simultaneously contributing to the shaping of these communities. [3] In the continuing process of learning how to do research, students are shaped by their school community, and they progressively learn what sources are acceptable to that community.

This emphasis on learning as process was also discussed by Jeff Maehre who argues that instructors who ban the use of Wikepedia in introductory research projects are sending their students conflicting signals. Maehre points out that college students, especially in their introductory classes, are in the process of learning to do research. Just as instructors encourage students to revise and develop their writing skills; so too should students be allowed to move from familiar skills in researching to more refined skills as their paper progress through the researching process. The ultimate goal should be students thinking individually and evaluating independently. Maehre believes that allowing students the freedom to do their own thinking and to form their own judgments is at the crux of the debate over Wikipedia. He contends that, "our policies toward this revolutionary information source apply some of the most profound pedagogical values we bring to the classroom." [1]

\section{HIGHER EDUCATION STUDENT RESEARCH METHODS}

Sudin and Franke set up a study in Sweden of upper secondary students, their teachers, and the librarians. All were encouraged to participate in independent multidisciplinary research with no restrictions on Internet research or Wikipedia. The pupils and staff were observed for information gathering and judgments on a source's credibility. Students were 
also asked to blog about their research and explain why they chose some sources and rejected others. The authors delineated five themes from their research that applied to this group of students as they did their research.

The first clear theme in Sudin and Franke's study was that there was a definite link between the Google search engine and Wikipedia. Even though a few students went to the library for information over fourfifths of the students typed in Google as their first step in research. Sudin and Franke point out that "the OCLU (2006) reports that among college students, as many as $89 \%$ begin their information search with a search engine. [3] Wikipedia is almost always at the top of the list in these searches. The researchers did find that students were aware of Wikipedia's shortcomings and credibility issues. Many students spoke of starting with this source to find more reliable links. In interviews with the students as they researched, it was evident that they were using Wikipedia "strategically" [3] The researchers also found that even though Wikipedia was not expressly prohibited in this particular project, the students had learned in the school community that Wikipedia was not viewed as a source for citation, and they sought other sources for credible authority.

A second theme found in the study was that students were developing a sense of how knowledge is formed. Students did realize that Wikipedia was collaborative and they had an overall respect for that process; however, students and teachers were not always aware of the architecture of Wikipedia and the features of Wikipedia that offer a discussion page and past edits. The researchers found that students were not very advanced in using the features of digital technology which could help them to verify information, uncover controversies, and read counter opinions. In addition, the teachers and librarians did not offer this information to students as they worked through the research process. As a community of learners it appears that both faculty and students need to learn to navigate the web in a more complete and sophisticated fashion.

Volume XI, No. 1, 2010
A third theme in the study involved the judgments students made about the trustworthiness of the author or organization that produced the website. Respected organizations and government publications were the most trusted by the students. The authors felt that democratic principles and the operations of a free press were involved in this perception. The students often needed assistance in understanding which organizations to trust. This was especially true if they were studying sites from other countries where they did not know the history and culture of the organizations.

A fourth theme evident in this study was that students preferred to work on the Internet and a few never used any print sources for their projects. However, students continue to perceive print sources as more reliable than Internet sites. It was again evident that students had been trained to question their Internet sources but not trained in how to use the tools and architecture of Internet websites to check for reliability.

The final theme delineated by the researchers was that students judged the credibility of their research by the genre of the tool they used. Students felt that blogs contained opinions which were often unsubstantiated by facts. Published books were at the opposite end of the continuum and judged as the most reliable and the most factual. Wikipedia was judged to be not as reliable because it is collaborative and subject to change, even though the genre reads like a published encyclopedia. Students seem to use Wikipedia in two distinct ways. They trust the source for quick information outside of the school, and it is often the first source they will use for research. Yet students still maintain a skeptical stance when they use Wikipedia to research a school project.

Sudin and Franke conclude that educational practices in gathering information for research will continue to be influenced by digital media. It is now even more important for students to learn how to navigate sites like Wikipedia and to use Internet resources effectively. Students can be taught to refer to the history of a source and to understand the technology available to them to authenticate research on the
Issues in Information Systems 
Internet. Schools have an obligation to update their teaching on the reliability of sources and to include more hands on practice in utilizing digital information technologies to their full extent.

\section{IMPLICATIONS FOR TEACHING RESEARCH IN HIGHER EDUCATION}

The controversy over Wikipedia in college research brings many of the traditional judgments educators make about sources into question. Brett Potash feels that this issue is rooted into the view many have of the nature of knowledge. [2] $\mathrm{He}$ argues that Wikipedia could be seen as the ultimate experiment in the democratization of knowledge.

Potash contends that many of the best practices of research gathering can be seen in the Wikipedia process. "The work is meaningful, it has a practical purpose, and it utilizes different forms of media" [2]

\section{CONCLUSIONS}

In reviewing the literature on the use of Wikipedia in upper high school and college level research, it is clear that the great revolution in information technology may mean changes are needed in the college classroom. Students will continue to learn how to judge sources for reliability. They will become familiar with college library print sources and respected journals in their fields. Their research writing and information gathering skill will be revised and refined as they progress in their studies. Many of the same reliability tests and standards will be transmitted to them during the research process. However, there is also a clear need for instructors to update their knowledge and skills in using Internet resources fully.

Information technologies such as Wikipedia need not be banned or discredited. They need to be seen as yet another important tool in information gathering. These sources will evolve and challenge colleges to remain current with the architecture and tabs which can lead to a check of the sources for an article and its history. Wikipedia itself could become an important part of helping students move to the complex and critical research skills colleges require. An added benefit in changing the negative attitude that many instructors have toward the Internet and Wikipedia is that there will be a respect for the knowledge and skills students are already bringing with them to their college studies. They will be able to move from more familiar sources to more traditional ones more confidently. Instructors at the higher education level will be more effective once they have broadened their knowledge and use of the Internet as a source for knowledge and they give up the preconceived notion that Internet sources are so inferior to traditional source. Students will then receive the skills they need to navigate the Internet's complexities and fully utilize it as a resource.

\section{REFERENCES}

1. Maehre, J. (2009). What It Means to Ban Wikipedia:an Exploration of the Pedagogical Principles at Stake. College Teaching, 57(4), 229-36. Retrieved June 21, 2010, from the ERIC database.

2. Potash, B. (2010, Spring). We Live in a Wiki World; Should our Students. independent school, 69,3 .

3. Sundin, O., \& Francke, H. (2009). In search of credibility: pupils' information practices in learning environments. Information Research, 14(4). Retrieved June 21, 2010, from http://InformationR.net/ir/14-4/paper418.html

4. Wikipedia - Wikipedia, the free encyclopedia. Retrieved June 21, 2010, from http://en.wikipedia.org/wiki/Wikipedia 